\title{
Passing the Barrier - How Computer Simulations Can Help to Understand and Improve the Passive Membrane Permeability of Cyclic Peptides
}

\author{
Stephanie M. Linker, Shuzhe Wang, Benjamin Ries, Thomas Stadelmann, and Sereina Riniker ${ }^{\star}$
}

\begin{abstract}
Proteins with large and flat binding sites as well as protein-protein interactions are considered "undruggable' with conventional small-molecule drugs. Cyclic peptides have been found to be capable of binding to such targets with high affinity, making this class of compounds an interesting source for possible therapeutics. However, the oftentimes poor passive membrane permeability of cyclic peptides still imposes restrictions on the applicability of cyclic peptide drugs. Here, we describe how computational methods in combination with experimental data can be used to improve our understanding of the structure-permeability relationship. Especially the conformational dynamic and chameleonic nature of cyclic peptides, which we investigate by a combination of MD simulations and kinetic modeling, is important for their ability to permeate passively through the membrane. The insights from such studies may enable the formulation of design principles for the rational design of permeable cyclic peptides.
\end{abstract}

Keywords: Cyclic peptides $\cdot$ Kinetic modelling $\cdot$ Membrane permeability $\cdot$ Molecular dynamics

\section{Introduction}

Studies based on the sequence and structural similarity of the human genome estimate that traditional small-molecule drugs can only target $10-15 \%$ of human genes.[1] Small-molecule drugs primarily bind to deep and relatively narrow binding pockets. ${ }^{[2]}$ Many potential targets such as protein-protein interfaces or class B G-protein coupled receptors (GPCRs) have, however, large and flat binding sites and are thus considered 'difficult-todrug'. To address this issue, increasing efforts in pharmaceutical research focus on the discovery of new compounds beyond small-molecule drugs. Cyclic peptides (and macrocycles in general) have been shown to be capable of binding to large and flat binding sites with high affinity, ${ }^{[3]}$ making them possible therapeutics for difficult targets. In addition, cyclic peptides display low toxicity due to their amino acid makeup. ${ }^{[4]}$ Although first cyclic peptide drugs - like the immunosuppressive cyclosporin $\mathrm{A}^{[5]}$ or the anti-cancer drug romidepsin${ }^{[6]}$ - are already on the market, more insights into the mode of action and pharmacokinetics of cyclic peptides are needed.

The therapeutic application of cyclic peptides is often limited by their low cell permeability and oral bioavailability. ${ }^{[7]}$ In order to reach intracellular targets, cyclic peptides need to cross the cell membrane barrier. Although cyclic peptides show an improved membrane permeability in comparison to their linear counterparts, most cyclic peptides are still not permeable enough for oral administration. ${ }^{[7]}$ In general, passive permeability decreases with increasing size of the peptide. ${ }^{[8]}$ In this article, we focus on cyclic peptides that are small enough to potentially cross membranes passively. A prominent example is the natural product cyclosporin $\mathrm{A},{ }^{[5,9]}$ which has a medium to good membrane permeability. Both experimental and computational efforts aim to learn from the known examples, understand the structure-permeability relationship, and derive design principles for bioavailable cyclic peptides.

The predominant strategy to distinguish permeable and impermeable compounds is to synthesize a series of cyclic peptides and experimentally determine their membrane permeability. Typical approaches to improve the permeability in a given compound series are to investigate different backbone $N$-methylation patterns, side chain modifications, and stereochemical changes. ${ }^{[10]}$ In some cases, the addition of a cell-penetrating peptide that triggers endocytosis was found to improve bioavailability. ${ }^{[1]}$ However, these changes are often applied in a time-consuming trial-and-error process and improvements in permeability may come with loss in binding affinity. Therefore, demands and efforts have increased to develop computational models to rationalize membrane permeability and support the development of bioavailable cyclic peptide drugs.

\section{Computational Approaches for Cyclic Peptides}

Calculation of the membrane permeability of small molecules ranges back to Meyer and Overton. ${ }^{[2]}$ The calculated lipophilicity measure $\mathrm{Alog} \mathrm{P}^{[13]}$ is often used as a heuristic for the passive permeability of drug-like molecules. However, such simple cheminformatics models based on the 2D topology of molecules do not apply to the more complex cyclic peptides, where properties become conformation-dependent. This conformational flexibility has been found to be crucial for permeability. ${ }^{[9,14-17]}$ Indeed, the relationship between size and permeability differs distinctly between small molecules and macrocycles, an effect that cannot be explained with traditional permeation models. ${ }^{[8]}$ As a result, the focus shifted to computational methods that can take into account the flexibility of molecules. 


\subsection{Molecular Dynamics Simulations of Cyclic Peptides}

Molecular dynamics (MD) simulations use Newtonian physics to simulate the motion and interaction of molecules. They are a widely used tool to study kinetics, energetics, and conformations of biological and chemical systems (for a review see e.g. ref. [18]). Using MD, the dynamics of cyclic peptides can be analyzed in atomic detail, a resolution that cannot be achieved with experimental techniques. The hypotheses generated based on the simulations are then tested and validated in experiments. Therefore, it is common practice to combine MD simulations and experimental techniques to obtain a holistic picture.

Combinations of NMR and MD studies revealed that cyclic peptides display a chameleonic behavior. ${ }^{[9,19-21]}$ Cyclic peptides can form intramolecular hydrogen bonds, leading to a so-called closed conformation, where most polar groups are shielded from the apolar environment. Thanks to the conformational flexibility, cyclic peptides can also adopt open conformations forming hydrogen bonds with surrounding water molecules (Fig. 1).

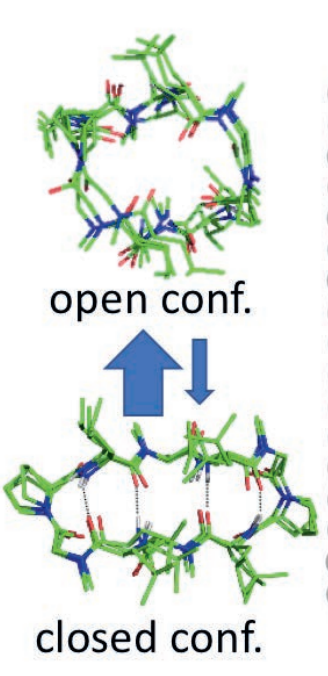

extracellular

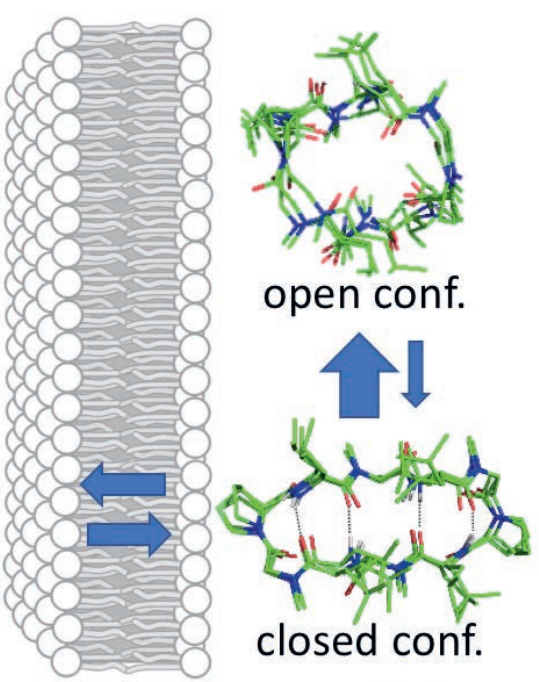

intracellular
Fig. 1. Model for the passive membrane permeability of cyclic peptides. ${ }^{[9,14]}$ In solution, cyclic peptides are assumed to adopt predominantly open conformations, forming hydrogen bonds with the solvent. The closed conformation is characterized by intramolecular transannular hydrogen bonds, which shield the polar groups from an apolar environment. This conformational flexibility is thought to make cyclic peptides membrane permeable.

Due to the shielding of polar groups in the closed conformation, the peptide has a lower desolvation energy barrier for moving into the membrane. Therefore, the closed conformation (also termed low-permittivity conformation ${ }^{[14]}$ ) is considered the main permeable 'species'. ${ }^{[14]}$ However, the existence of the closed conformation is a necessary but not sufficient condition for permeability. ${ }^{[17]}$ Other properties like side chains, size, and the polar or hydrophobic surface area heavily influence the permeability of cyclic peptides. A recent study combining MD simulations, NMR and permeability assays showed that the size of the largest connected hydrophobic surface of the cyclic peptide is decisive for its permeability.[22] To increase the membrane permeability of cyclic peptides, the authors suggest to place $N$-methylations or hydrophobic residues in positions where they connect two separated hydrophobic surfaces. Recent work in our group revealed how a change in only a single stereocenter in two semi-peptidic macrocycles (Fig. 2) leads to a 'permeability cliff' in experimental permeability assays. ${ }^{[23]}$ Using a combination of NMR and MD studies, we could show how the change affects the conformational ensemble of the macrocycles and through this the population of conformations with a maximal number of intramolecular hydrogen bonds in the apolar (chloroform) environment.

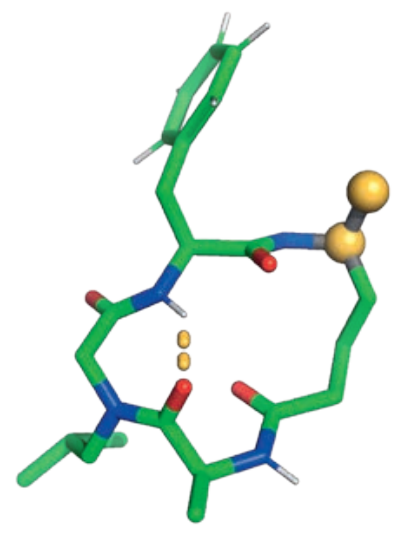

Nleu $-5 S$

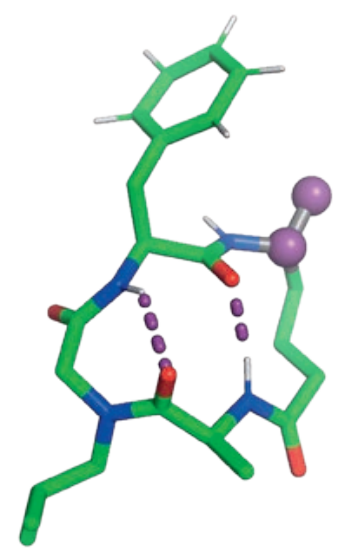

Nleu $-5 R$
Fig. 2. Two semi-peptidic macrocycles, Nleu-5S and Nleu-5R, differ only in the stereochemistry of the methyl group in the alkyl linker (golden and purple spheres). The shown structures are snapshots from our extensive MD simulations representing the differences in the hydrogen-bond patterns of the two compounds in apolar environments. ${ }^{[23]}$

The findings described in these studies would not have been possible without the atomistic insights on the cyclic peptides provided by MD simulations. Furthermore, computer simulations may not only help to improve the permeability, but also to identify peptide candidates for specific targets. For example, MD simulations have been used to pre-select cyclic peptides drug candidates for targeting protein-protein interfaces. ${ }^{[24]}$

\subsection{Kinetic Models of Cyclic Peptides}

As discussed above, conformational flexibility of cyclic peptides is a crucial determinant for their membrane permeability. Kinetic information is needed to fully account for the dynamics of cyclic peptides. In contrast to many other computational techniques, MD simulations can provide such kinetic information. The MD procedure we have developed for permeability analysis is shown in Fig. 3. ${ }^{[21,25]}$ First, a diverse conformer set is generated for a cyclic peptide of interest. When an experimental structure (X-ray or NMR) is available, this can be done by taking snapshots from an enhanced sampling simulation starting from the experimental structure. Alternatively, in silico conformer generators can be used. Due to their size, cyclic peptides span a far larger conformational space than can be exhaustively sampled. It is therefore instrumental to bias sampling towards the more relevant phase space. We recently improved the RDKit conformer generator ETKDG $^{[26]}$ specifically for macrocyclic species by incorporating experimental torsion information as well as shape and interaction heuristics. ${ }^{[27]}$ Once selected, each conformer is solvated in water (mimicking the polar extracellular condition) and chloroform (mimicking the apolar dielectric environment within the lipid core). Next, extensive simulations are performed in parallel in the two solvents, starting from the conformer set. The individual simulations are typically on the order of hundreds of nanoseconds and total sampling time on the order of tens of microseconds per solvent. The parallel trajectories are used to build Markov state models (MSMs). ${ }^{[28-31]}$ MSMs extract metastable conformational states and the interconversion rate between them, and enable the comparison of the conformational behavior of structurally similar compounds. They are therefore a valuable tool to rationalize 


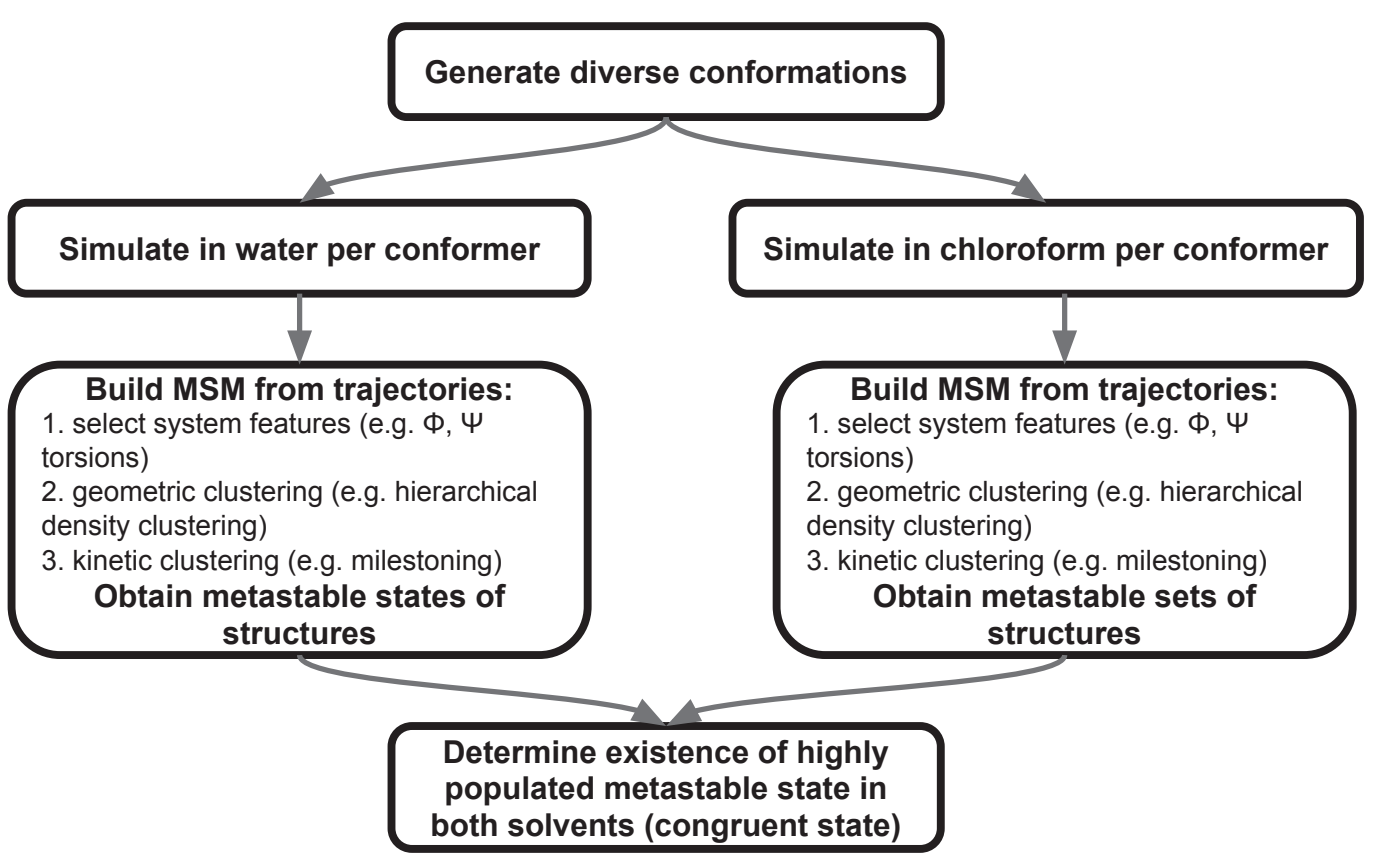

Fig. 3. Schematic illustration of our workflow to determine congruent states from extensive MD simulation and Markov state models (MSMs). ${ }^{25]}$ membrane permeability. The kinetic models obtained can also be used to compare internal flexibility between slowly exchanging core conformations with experimentally determined NMR $\mathrm{T}_{2}$ relaxation times. ${ }^{[32]}$ When constructing MSMs, we use densitybased clustering algorithms.[21] Recently, we could show that a hierarchical density-based clustering is able to better capture metastable states residing at free energy wells of varied depth. ${ }^{[33]}$

Our kinetic models based on MD simulations of the permeable cyclosporin A revealed the existence of so-called congruent conformational states, ${ }^{[9]}$ which are populated in both polar and apolar environments. Such congruent conformational states can facilitate the transition between different environments and therefore increase permeability (Fig. 1). The closed conformation of cyclosporin A is an example of a congruent conformation. In addition, MSMs suggested that the main difference between cyclosporin A and the structurally closely related but impermeable cyclosporin $\mathrm{E}$ are the interconversion rates between their metastable states. ${ }^{[20]}$ When applying our workflow to rationalize the membrane permeability differences of six cyclic decapeptides sharing the same backbone $N$-methylation pattern but differing in their side chain composition, ${ }^{[21]}$ the kinetic models revealed that the population of the closed conformational state in water correlates with the passive membrane permeability. Based on this observation, we expanded our analysis onto a larger set of 24 decapeptides, systematically covering side chain compositions. We find that the closed state population in water remains a distinguishing metric of passive permeability and the MD simulations provided mechanistic insights into the formation of the closed conformation. ${ }^{[25]}$

\subsection{Simulations of Cyclic Peptides at Membranes and Interfaces}

To better understand the passive membrane permeability of cyclic peptides, it would be desirable to directly simulate their interaction with lipid membranes. However, these large and complex systems require tremendous computational resources. Studies investigating these interactions for passively permeating peptides are therefore still rare, and the few available works involved computational tricks to speed up the simulations. ${ }^{[34]}$ For example, Wang et al..$^{[34]}$ simulated cyclosporin A at a POPC lipid bilayer by increasing the flexibility of the peptide backbone by $40 \%$ and increasing the temperature to over $400 \mathrm{~K}$. At such elevated temperatures, the membrane in the simulation becomes porous. Nevertheless, their observations supported the view that confor- mational flexibility is important for membrane permeability and the authors hypothesized that a higher flexibility of the peptide leads to a higher permeability. Simulations of unbiased systems at room temperature systems are needed to gain insights into a physically realistic permeation process. Our research is currently aiming to fill this gap. Preliminary results suggest that efforts to optimize permeability should not only focus on the properties of cyclic peptides in solvents but also consider the peptide's interactions with interfaces.

\section{Conclusions and Outlook}

MD simulations yield atomistic insights into the conformational dynamics of cyclic peptides. Together with experimental efforts, the findings have already helped to improve our understanding of the passive membrane permeability of cyclic peptides. Future research will focus on the role of the membrane in the permeation process and on further unveiling the complex relationship between structure and conformational behavior of cyclic peptides. This knowledge will help to improve the rational design of peptidic drug candidates and the development of novel therapeutics for currently undruggable targets.

\section{Acknowledgements}

The authors gratefully acknowledge financial support by the Swiss National Science Foundation (Grant number 200021-178762) and the Scholarship Fund of the Swiss Chemical Industry. S.M.L was supported by the PhD scholarship of the German National Academic Foundation.

Received: April 15, 2021

[1] A. L. Hopkins, C. R. Groom, Nat. Rev. Drug Discov. 2002, 1, 727, https://doi.org/10.1038/nrd892

[2] G. Caron, J. Kihlberg, G. Goetz, E. Ratkova, V. Poongavanam, G. Ermondi, ACS Med. Chem. Lett. 2021, 12, 13, https://doi.org/10.1021/acsmedchemlett.0c00581

[3] B. C. Doak, J. Zheng, D. Dobritzsch, J. Kihlberg, J. Med. Chem. 2016, 59, 2312, https://doi.org/10.1021/acs.jmedchem.5b01286

[4] A. Zorzi, K. Deyle, C. Heinis, Curr. Opin. Chem. Biol. 2017, 38, 24, https://doi.org/10.1016/j.cbpa.2017.02.006

[5] A. Rüegger, M. Kuhn, H. Lichti, H.-R. Loosli, R. Huguenin, C Quiquerez, A. von Wartburg, Helv. Chim. Acta 1976, 59, 1075, https://doi.org/10.1002/hlca.19760590412

[6] H. Nakajima, Y. B. Kim, H. Terano, M. Yoshida, S. Horinouchi, Exp. Cell Res. 1998, 241, 126, https://doi.org/10.1006/excr.1998.4027 
[7] C. Adessi, C. Soto, Curr. Med. Chem. 2002, 9, 963, https://doi.org/10.2174/0929867024606731

[8] C. R. Pye, W. M. Hewitt, J. Schwochert, T. D. Haddad, C. E. Townsend, L. Etienne, Y. Lao, C. Limberakis, A. Furukawa, A. M. Mathiowetz, D. A. Price, S. Liras, R. S. Lokey, J. Med. Chem. 2017, 60, 1665, https://doi.org/10.1021/acs.jmedchem.6b01483

[9] J. Witek, B. G. Keller, M. Blatter, A. Meissner, T. Wagner, S. Riniker, J. Chem. Inf. Model. 2016, 56, 1547, https://doi.org/10.1021/acs.jcim.6b00251

[10] L. K. Buckton, M. N. Rahimi, S. R. McAlpine, Chem. - A Eur. J. 2021, 27 , 1487, https://doi.org/10.1002/chem.201905385

[11] M. Kristensen, D. Birch, H. M. Nielsen, Int. J. Mol. Sci. 2016, 17, 185 , https://doi.org/10.3390/ijms17020185.

[12] C. E. Overton, 'Studien Über Die Narkose. Zugleich Ein Beitrag Zur Allgemeinen Pharmakologie', Gustav Fischer, Jena, 1901.

[13] A. K. Ghose, V. N. Viswanadhan, J. J. Wendoloski, J. Phys. Chem. A 1998, 102, 3762, https://doi.org/10.1021/jp980230o

[14] T. Rezai, J. E. Bock, M. V. Zhou, C. Kalyanaraman, R. S. Lokey, M. P. Jacobson, J. Am. Chem. Soc. 2006, 128, 14073, https://doi.org/10.1021/ja063076p

[15] T. Rezai, B. Yu, G. L. Millhauser, M. P. Jacobson, R. S. Lokey, J. Am. Chem. Soc. 2006, 128, 2510, https://doi.org/10.1021/ja0563455

[16] P. Matsson, J. Kihlberg, J. Med. Chem. 2017, 60, 1662, https://doi. org/10.1021/acs.jmedchem.7b00237

[17] S. Riniker, Future Med. Chem. 2019, 11, 637, https://doi.org/10.4155/fmc-2018-0429

[18] R. O. Dror, R. M. Dirks, J.P. Grossman, H. Xu, D. E. Shaw, Annu. Rev. Biophys. 2012, 41, 429, https://doi.org/10.1146/annurev-biophys-042910-155245

[19] A. Alex, D. S. Millan, M. Perez, F. Wakenhut, G. A. Whitlock, MedChemComm 2011, 2, 669, https://doi.org/10.1039/C1MD00093D

[20] J. Witek, M. Mühlbauer, B. G. Keller, M. Blatter, A. Meissner, T. Wagner, S. Riniker, ChemPhysChem 2017, 18, 3309, https://doi.org/10.1002/cphc.201700995

[21] J. Witek, S. Wang, B. Schroeder, R. Lingwood, A. Dounas, H.-J. Roth, M. Fouché, M. Blatter, O. Lemke, B. Keller, S. Riniker, J. Chem. Inf. Model. 2019, 59, 294, https://doi.org/10.1021/acs.jcim.8b00485

[22] H. N. Hoang, T. A. Hill, D. P. Fairlie, Angew. Chem. Int. Ed. 2021, 60, 8385, https://doi.org/10.1002/anie.202012643

[23] C. Comeau, B. Ries, T. Stadelmann, J. Tremblay, S. Poulet, U. Fröhlich, J. Côté, P.-L. Boudreault, R. M. Derbali, P. Sarret, M
Grandbois, G. Leclair, S. Riniker, É. Marsault, J. Med. Chem. 2021 , https://doi.org/10.1021/acs.jmedchem.0c02036.

[24] B. L. Santini, M. Zacharias, Front. Chem. 2020, 8, 573259 , https://doi.org/10.3389/fchem.2020.573259

[25] S. Wang, G. König, H.-J. Roth, M. Fouché, S. Rodde, S. Riniker, J. Med. Chem. 2021, submitted.

[26] S. Riniker, G. A. Landrum, J. Chem. Inf. Model. 2015, 55, 2562, https://doi.org/10.1021/acs.jcim.5b00654.

[27] S. Wang, J. Witek, G. A. Landrum, S. Riniker, J. Chem. Inf. Model. 2020, 60 , 2044, https://doi.org/10.1021/acs.jcim.0c00025

[28] C. Schütte, A. Fischer, W. Huisinga, P. Deuflhard, J. Comput. Phys. 1999, 151, 146, https://doi.org/10.1006/jcph.1999.6231

[29] W. C. Swope, J. W. Pitera, F. Suits, J. Phys. Chem. B 2004, 108, 6571, https://doi.org/10.1021/jp037421y

[30] V. S. Pande, K. Beauchamp, G. R. Bowman, Methods 2010, 52, 99, https://doi.org/10.1016/j.ymeth.2010.06.002

[31] J. H. Prinz, H. Wu, M. Sarich, B. Keller, M. Senne, M. Held, J. D. Chodera, C. Schtte, F. Noé, J. Chem. Phys. 2011, 134, 174105, https://doi.org/10.1063/1.3565032

[32] T. Stadelmann, G. Subramanian, S. Menon, C. E. Townsend, R. S Lokey, M. O. Ebert, S. Riniker, Org. Biomol. Chem. 2020, 18, 7110, https://doi.org/10.1039/D0OB01447H

[33] R. G. Weiß, B. Ries, S. Wang, S. Riniker, J. Chem. Phys. 2021, 154, 084106 , http://doi.org/10.1063/5.0025797

[34] C. K. Wang, J. E. Swedberg, P. J. Harvey, Q. Kaas, D. J. Craik, J. Phys Chem. B 2018, 122, 2261, https://doi.org/10.1021/acs.jpcb.7b12419

\section{License and Terms}

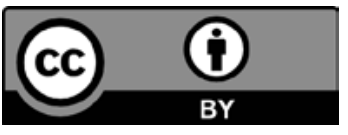

This is an Open Access article under the terms of the Creative Commons Attribution License CC BY 4.0. The material may not be used for commercial purposes.

The license is subject to the CHIMIA terms and conditions: (http:// chimia.ch/component/sppagebuilder/?view = page \&id $=12$ ).

The definitive version of this article is the electronic one that can be found at https://doi.org/10.2533/chimia.2021.518 